\title{
Superatom-assembly induced transition from insulator to semiconductor. A theoretical study
}

\author{
Jia Wang ${ }^{1,2}$, Wanrun Jiang ${ }^{1,2}$, Weiyu Xie ${ }^{1,2}$, Jianpeng Wang ${ }^{1,2}$ and Zhigang Wang ${ }^{1,2^{*}}$
}

\begin{abstract}
Assembly is an effective way to realize the functionalization potential of boron-based superatoms. Here we study the interaction between typical boron-based $B_{40}$ superatoms using the density functional theory. Our results reveal that different oligomers constructed by modulating the arrangement of two $B_{40}$ superatoms still retain some of the superatomic properties associated with their monomeric form despite possessing different electronic structures. While the inner shell superatomic orbitals maintain their electronic localization, the valence shell superatomic orbitals cannot maintain their original shape due to bonding and antibonding hybridization. Furthermore, the decreasing of band gap means that the $B_{40}$ oligomers could achieve a transformation from insulators to semiconductors. The decreased band gap is possibly due to the disappearance of the superatomic orbitals with the principal quantum number of two. Our findings highlight that superatom-superatom interactions could induce synergy effects that differ from their monomers. Therefore, this research will aid in the development of new materials and devices that are constructed from superatoms.
\end{abstract}

Keywords: superatom, intermolecular interaction, electronic structure, density functional theory

\section{INTRODUCTION}

Cluster-assembled materials have attracted increasing attention due to their potential applications in molecular recognition, molecular electronics, bio-labeling and sensing, biomedicine, and catalysis [1-8]. In addition, superatom-assembled materials are being designed for novel optical and electromagnetic functionalities based on $\mathrm{C}_{60}[9,10], \mathrm{Al}_{13}{ }^{-}$[11-13], $\mathrm{As}_{7}$ [14], or $\mathrm{Au} / \mathrm{Ag}$ nanoparticle $[15,16]$ building blocks, as the understanding about superatoms becomes increasing proficient. Recent studies have demonstrated that the $B_{40}$ cage exhibits a superatomic property $[17,18]$. Its stability has been confirmed using photoelectron spectroscopy, and its high-temperature structural robustness has also been predicted through molecular dynamics simulations [19]. Therefore, we consider whether $\mathrm{B}_{40}$ can replace $\mathrm{C}_{60}$ as a superatom building block for assembled materials, and try to obtain a better understanding of its assembly behavior from a superatomic perspective.

A series of studies have been undertaken since $B_{40}$ was first synthesized to better understand the doping properties of this structure [20-22] because it possesses a slightly smaller radius than $C_{60}$ [19]. Related structures have demonstrated promising potential in nanodevice applications. It has been reported that $\mathrm{B}_{40}$ fullerene could be an efficient nanomaterial for $\mathrm{CO}_{2}$ capture $[23,24]$ or hydrogen storage [25]. $\mathrm{B}_{40}$ can also be used as a highsensitivity molecular device [26], molecular rectifier, and photodetector [27]. Furthermore, $\mathrm{B}_{40}$ fullerene can interact with alkali metals or superalkali $\mathrm{M}_{3} \mathrm{O}(\mathrm{M}=\mathrm{Li}$ and $\mathrm{K})$ to enhance the first hyperpolarizability and be introduced as a nonlinear optical material $[28,29]$. In addition, the intermolecular interaction properties between $\mathrm{B}_{40}$ monomers have been reported to study the $\mathrm{B}_{40}$ cluster reactivity [30]. We first proposed the superatomic characteristics of $B_{40}$ in 2017 [17], which expanded the superatom family and brought a new perspective on the utilization of the $B_{40}$ superatom as a functional element. We hypothesize that the two sets of $B_{40}$ superatomic orbitals may play different roles in the assembly process.

Unlike conventional or natural materials that are composed of atoms and molecules, cluster assembly enables us to design novel materials using clusters as the building blocks, possessing new properties and functions [31]. Therefore, we studied the interactions between $B_{40}$ superatoms to realize the materials assembled from boron-based superatoms. Our results show that there are two sets of $B_{40}$ superatomic orbitals, with one playing a role in

\footnotetext{
${ }^{1}$ Institute of Atomic and Molecular Physics, Jilin University, Changchun 130012, China

${ }^{2}$ Jilin Provincial Key Laboratory of Applied Atomic and Molecular Spectroscopy (Jilin University), Changchun 130012, China

* Corresponding author (emails: wangzg@jlu.edu.cn or wangzg1978@hotmail.com)
} 
maintaining $\mathrm{B}_{40}$ superatomic stability of its monomeric form and the other playing a role in superatomic interactions and connections. Furthermore, the oligomer has a lower band gap compared to the $\mathrm{B}_{40}$ monomer, allowing the oligomer to transit from insulator to a semiconductor. Therefore, we propose a possible route to synthesize cluster-assembled materials using $B_{40}$ superatoms.

\section{COMPUTATIONAL METHODS}

The empirical dispersion-corrected density functional theory was used to fully obtain the intermolecular interaction of $\mathrm{B}_{40}$ structures [32]. The hybrid generalized gradient approximation (hybrid GGA) was employed at the PBE0-D3/6-31G* level [33-35]. Previous studies have extensively tested the PBE0 functional and found it to be suitable for boron clusters $[19,36]$. In addition, the frequency analyses were conducted using the PBE0-D3/6$31 \mathrm{G}^{*}$ level of theory to characterize the nature of the stationary points, and all the optimized structures were confirmed to be local minima. The Gaussian09 software package was used to carry out all the computations mentioned above [37]. Energy decomposition analysis was performed to understand interaction mechanism, following the schemes of Morokuma [38], and Ziegler and Rauk [39] in the ADF software package [40]. The
DMol3 software package was used to perform molecular dynamics simulations at the PBE/DND level [41]. The simulations were initiated from the equilibrium geometry, random velocities were assigned to the atoms, and the simulations were run for approximately $10 \mathrm{ps}$, with a time step of $1 \mathrm{fs}$.

\section{RESULTS AND DISCUSSION}

The $B_{40}$ cage has two hexagons $\left(6_{1}\right.$ and $\left.6_{2}\right)$ at the top and bottom, and four heptagons $\left(7_{1}, 7_{2}, 7_{3}\right.$, and $\left.7_{4}\right)$ that are evenly distributed around the waist, as the lower right corner of Fig. 1 shows. Here we enumerated various potential directions along the two $\mathrm{B}_{40}$ cages and obtained eight possible structures that formed via the interaction between two $B_{40}$ superatoms, arranged according to their total energies. When the $7_{3}$ heptagon of one cluster stacks onto the vertical $7_{1}$ heptagon of another (denoted as $7_{3} \perp 7_{1}$ ) (Fig. 1a), the conformation energy is the lowest. The conformation in Fig. $1 \mathrm{~b}$ is formed when the B atoms of one cluster stack onto the B atoms of another cluster (denoted as vertex-vertex). The conformation in Fig. 1c is formed by placing the $7_{3}$ heptagon of one cluster onto the antiparallel $7_{1}$ heptagon of another. The conformations in Fig. 1d-h are formed when the $7_{3}$ heptagon or $6_{1}$ hexagon of one cluster stacks onto the $6_{2}$ or $6_{1}$ hexagon of another.

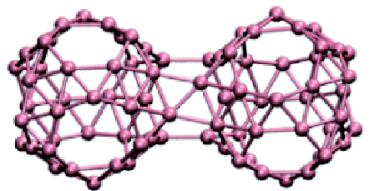

(a) $7_{3} \perp 7_{1}[0.0]$

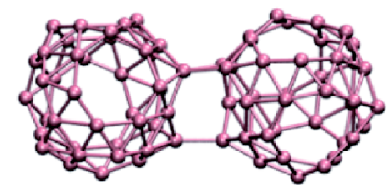

(d) $7_{3}-6_{2}[+0.34]$

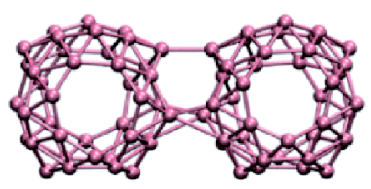

(g) $7_{3} \mu 7_{1}[+0.56]$

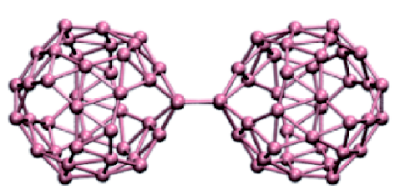

(b) vertex- vertex [+0.03]

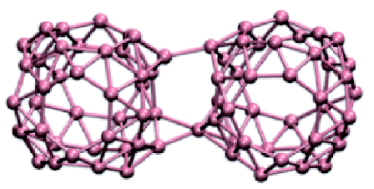

(e) $7_{3}-6_{1}[+0.42]$

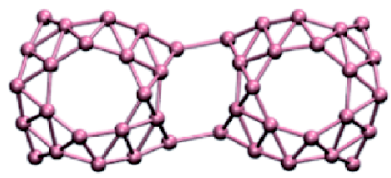

(h) $6_{1}-6_{1}[+0.80]$

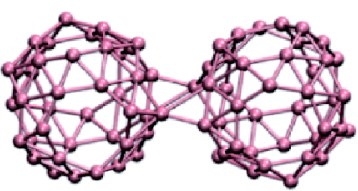

(c) $7_{3} / / 77_{1}[+0.26]$

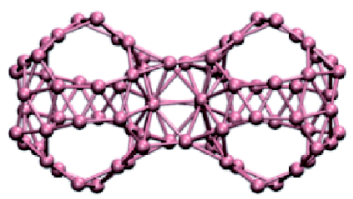

(f) $61-6_{2}[+0.46]$

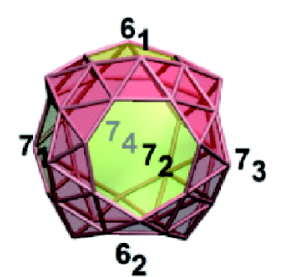

Figure 1 Structure diagrams for all the isomers formed via the interaction between two $\mathrm{B}_{40}$ superatoms. (a) Stacking the $7_{3}$ heptagon of one cluster onto the vertical $7_{1}$ heptagon of another. (b) Connecting the B atoms of one cluster with the B atoms of another cluster. (c) Placing the $7_{3}$ heptagon of one cluster onto the antiparallel $7_{1}$ heptagon of another. (d-h) Stacking the $7_{3}$ heptagon or $6_{1}$ hexagon of one cluster onto the $6_{2}$ or $6_{1}$ hexagon of another. (g) Stacking the $7_{3}$ heptagon of one cluster onto the parallel $7_{1}$ heptagon of another. The values in brackets are the relative energies between each isomers and the lowest energy structure, given in $\mathrm{eV}$. The structure of the $\mathrm{B}_{40}$ cage is shown in the lower right corner. The similar drawing method refers to Ref. [30]. 

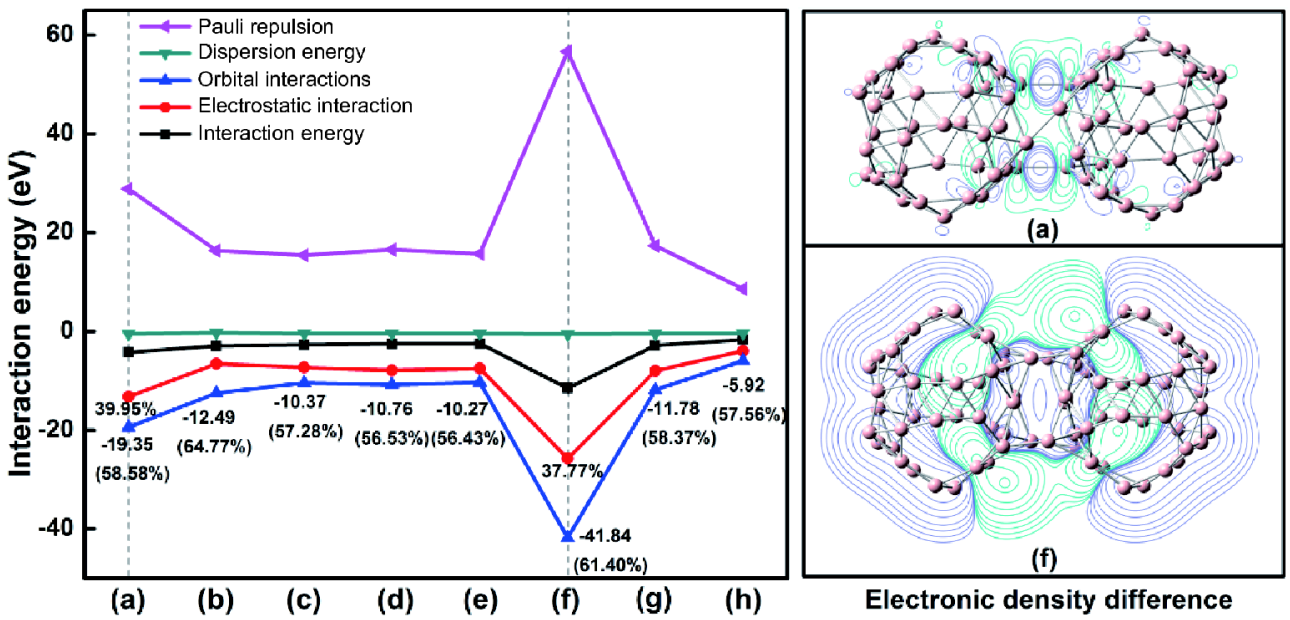

Electronic density difference

Figure 2 Energy decomposition (left panel) and electronic density difference (right panel). (a-h) represent the eight isomers in Fig. 1. The positive and negative values in the figure represent repulsion and attraction, respectively. The purple and blue lines in the right panels indicate electron accumulation and dissipation, respectively. Isovalue $=0.001$ a.u.

The conformation in Fig. $1 \mathrm{~g}$ is formed by placing the $7_{3}$ heptagon of one cluster onto the parallel $7_{1}$ heptagon of another. The relative energy between the lowest energy structure $\left(7_{3} \perp 7_{1}\right)$ and the other structures are shown in brackets. The total energy for each conformation is in Table S1 of the Supplementary information (SI). We also constructed the $\mathrm{B}_{40}$ trimer and tetramer based on the $7_{3} \perp 7_{1}$ conformation.

We analyzed the energy decomposition shown in the left panel of Fig. 2 to understand the interaction mechanism between the $B_{40}$ superatoms. See the SI for details on the energy decomposition analysis. The labels on the horizontal axis represent the eight isomers, ordered from left (lowest) to right (highest) according to their total energy. We can see that the interactions are dominated by the orbital term between two $\mathrm{B}_{40}$ superatoms. The orbital interaction of the $6_{1}-6_{2}$ conformation is the largest; however, that of $7_{3} \perp 7_{1}$ is also relatively large. This is because the two parts in $6_{1}-6_{2}$ are connected by four B$B$ atom pairs, with an average distance of approximately $1.71 \AA$ between two B atoms. This is shorter than those of the other isomers and, therefore, represents the strongest chemical bonding. It is noteworthy to mention that the $6_{1}-6_{2}$ interaction energy is the lowest among the eight isomers; however, its total energy is not the lowest. This highlights that the deformation of two $\mathrm{B}_{40}$ monomers in assembly is relatively severe due to the strong chemical bonding of the $6_{1}-6_{2}$ conformation. This results in an increase in deformation energy that compensates more of the energy decrease via electronic-structure bonding compared to the other cases, yielding the total energy order shown in Fig. 2. There is also a relatively strong chemical bonding between the two $B_{40}$ cages in $7_{3} \perp 7_{1}$. Furthermore, Pauli repulsion and electrostatic interaction are also larger for the $7_{3} \perp 7_{1}$ and $6_{1}-6_{2}$ conformations due to the shorter distance between the two superatoms. In addition, the dispersion interaction energy is small and has a negligible effect on the total interaction energy between $\mathrm{B}_{40}$ superatoms.

As the right panel of Fig. 2 shows, we analyzed the electron density differences of $7_{3} \perp 7_{1}$ and $6_{1}-6_{2}$ to better understand these conformations. The electron density of $7_{3} \perp 7_{1}$ indicates electron accumulation at the bonding region between the two monomers, with electron dissipation around this region. The electron density difference for $6_{1}-6_{2}$ indicates similar features but with stronger effects. The electron accumulation region is more delocalized within the contact region, and a large amount of electron dissipation forms in the outer sphere, as well as at two ends of $6_{1}-6_{2}$. This indicates the strong bonding in $6_{1}-6_{2}$ modulates its monomeric electronic structure more than in the other isomers.

We further analyzed the electronic structure of the $7_{3} \perp 7_{1}$ conformation since it had the lowest total energy. The molecular orbitals (MOs) of $7_{3} \perp 7_{1}$ are shown in Fig. 3 , with some MOs composed of $B_{40}$ monomer superatomic orbitals. This indicates that the $\mathrm{B}_{40}$ monomers retain some of their superatomic properties when they form $\mathrm{B}_{40}$ oligomers. There are two $1 \mathrm{~S}-1 \mathrm{~S}$ orbitals in the $7_{3} \perp 7_{1}$ MOs, which could be regarded as the in-phase and outof-phase cooperation between the 1S superatomic orbitals of the two monomers [17]. Similarly, there are six 1P-1P 


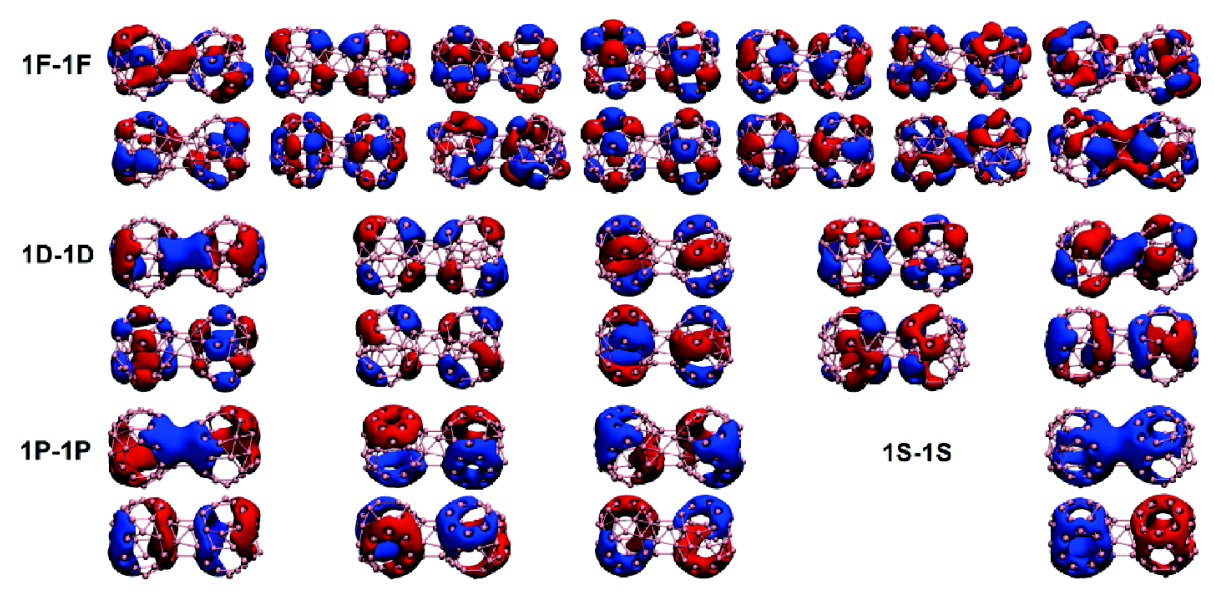

Figure $3 \mathrm{MOs}$ of the $7_{3} \perp 7_{1}$ conformation for the various $B_{40}$ superatomic orbital components. $1 \mathrm{~S}, 1 \mathrm{P}, 1 \mathrm{D}$, and $1 \mathrm{~F}$ refer to the $\mathrm{B}_{40}$ monomer superatomic orbitals. The blue and red areas indicate the positive and negative signs of the wave functions, respectively. Isovalue $=0.02$ a.u.

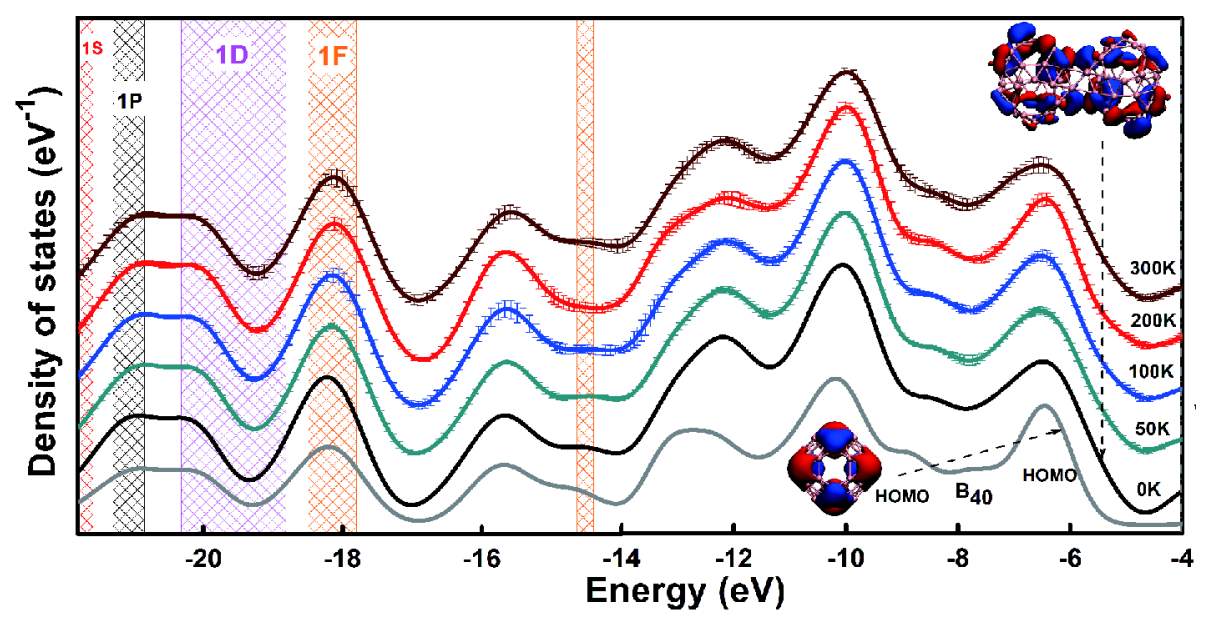

Figure 4 DOS temperature dependence for the $7_{3} \perp 7_{1}$ conformation. The red, black, pink, and orange hashed regions are the energy region corresponding to the $\mathrm{B}_{40}$ monomer $1 \mathrm{~S}, 1 \mathrm{P}, 1 \mathrm{D}$, and $1 \mathrm{~F}$ orbitals, respectively, in the $7_{3} \perp 7_{1}$ conformation at $0 \mathrm{~K}$. The two MOs in the figure are the HOMO for $\mathrm{B}_{40}$ and $7_{3} \perp 7_{1}$ conformation, respectively.

MOs and ten 1D-1D MOs, which are the in-phase and out-of-phase cooperation between the 1P and 1D orbitals, respectively, of the two $\mathrm{B}_{40}$ superatoms. There are seven double-occupied $1 \mathrm{~F}$ superatomic orbitals for single $B_{40}$ [17], meaning the $B_{40}$ dimer has fourteen double-occupied $1 \mathrm{~F}-1 \mathrm{~F}$ MOs, in accordance with the in-phase and out-of-phase cooperation between each other. A previous study found that $\mathrm{B}_{40}$ possesses $1 \mathrm{~S}, 1 \mathrm{P}, 1 \mathrm{D}$, and $1 \mathrm{~F}$ superatomic orbitals, as well as $2 \mathrm{~S}, 2 \mathrm{P}, 2 \mathrm{D}$, and $2 \mathrm{~F}$ superatomic orbitals with a higher orbital angular momentum [17]. Here we found that the monomeric superatomic orbitals were still qualitatively retained when the principal quantum number was one, whereas the orbitals disappeared when the principal quantum number was two due to the stronger orbital interaction. As Fig. 4 depicts, the monomer possesses a $2 \mathrm{~F}$ superatomic orbital as its highest occupied molecular orbital (HOMO) but no dimeric counterpart could be found in the electronic configuration of the dimer structure. The $7_{3} \perp 7_{1} \mathrm{HOMO}$ is also indicated in Fig. 4, which is analogous to the bonding of two atoms. The inner shell superatomic orbitals, 1S, $1 \mathrm{P}, 1 \mathrm{D}$, and $1 \mathrm{~F}$, retain their electronic localization when two superatoms are bonding; however, the valence shell superatomic orbitals, $2 \mathrm{~S}, 2 \mathrm{P}, 2 \mathrm{D}$, and $2 \mathrm{~F}$, cannot maintain their original orbital shape due to bonding and antibonding hybridization.

We also analyzed the density of state (DOS) for the eight isomers formed by the interaction between $\mathrm{B}_{40}$ superatoms, shown in Fig. S1, to explore the possibility of using $\mathrm{B}_{40}$ as a superatomic building block. The $\mathrm{B}_{40}$ dimer 
DOS trends are similar to the $\mathrm{B}_{40}$ monomer DOS trends, with the exception of the $6_{1}-6_{2}$ conformation, and their DOS values are twice as large as those of the $\mathrm{B}_{40}$ monomers. The reason that the $6_{1}-\sigma_{2}$ DOS trend is inconsistent with the other isomers may be due to the larger $6_{1}-6_{2}$ deformation energy and enhanced modulation of its superatom-based electronic structure.

We assembled a chain-like structure using $\mathrm{B}_{40}$ building blocks since one-dimensional nanowires have potential applications in molecular-based electronic devices. Molecular dynamics (MD) simulations were used to determine if the structures formed by the interaction between $B_{40}$ superatoms were stable under different temperatures. We simulated the MD of the $7_{3} \perp 7_{1}$ conformation in the $50-300 \mathrm{~K}$ temperature range, with intact and undamaged $7_{3} \perp 7_{1}$ conformation at $T=300 \mathrm{~K}$. Fig. S2 shows the typical geometric structures at different temperatures. Therefore, we consider that the $7_{3} \perp 7_{1}$ conformation is a stable structure. We then randomly selected ten structures in the MD and determined the average of their DOS for analysis, as shown in Fig. 4. The $7_{3} \perp 7_{1}$ DOS is almost the same as that of the $B_{40}$ monomer because the $B_{40}$ monomers in dimer maintain their superatomic nature. The MO regions dominated by the superatomic $1 \mathrm{~S}, 1 \mathrm{P}, 1 \mathrm{D}$, and $1 \mathrm{~F}$ orbitals of two $\mathrm{B}_{40}$ monomers are marked in Fig. 4. Furthermore, we found more obvious DOS fluctuations as the temperature increased. These enhanced DOS fluctuations occur because the structural changes become more apparent with increasing temperature. The temperature influence on $7_{3} \perp 7_{1}$ is small, even though both the structure and DOS vary with temperature. Therefore, we consider the geometric and electronic structures of the $7_{3} \perp 7_{1}$ conformation are stable.

We constructed $\mathrm{B}_{40}$ trimers and tetramers based on the $7_{3} \perp 7_{1}$ conformation (Fig. 5) to analyze the stability of the $\mathrm{B}_{40}$ superatomic assembly. The average $\mathrm{B}-\mathrm{B}$ bond length is approximately $1.70 \AA$ in these oligomers, smaller than the bulk case and indicates a stronger atomic interaction [42]. In addition, we analyzed their MOs to determine how well these oligomers maintain their superatomic character. As Figs S3 and S4 show, similar to the $B_{40}$ dimer, the $\mathrm{B}_{40}$ trimer and tetramer also have some MOs that are composed of the $\mathrm{B}_{40}$ superatomic $1 \mathrm{~S}, 1 \mathrm{P}, 1 \mathrm{D}$, and 1F orbitals. Furthermore, our results show that the gap between the HOMO and the lowest unoccupied molecular orbital (HOMO-LUMO) gap of the $\mathrm{B}_{40}$ monomer is $3.18 \mathrm{eV}$, and those of the dimer and trimer are 1.42 and $1.10 \mathrm{eV}$, respectively. The band gap decrease is likely due to the disappearance of superatomic orbitals with a

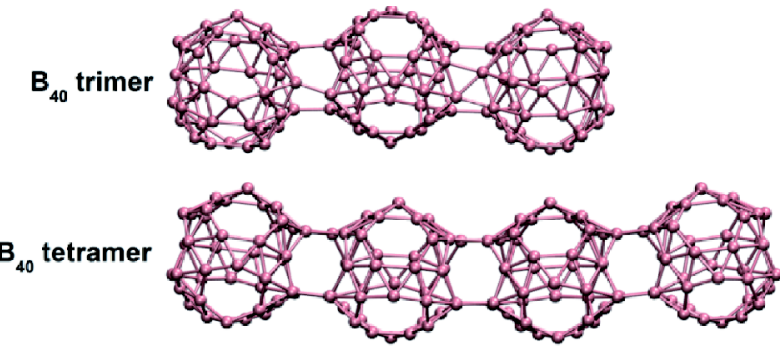

Figure $5 \mathrm{~B}_{40}$ trimer and $\mathrm{B}_{40}$ tetramer structures.

Table 1 HOMO-LUMO Gap for $\mathrm{B}_{40}$ oligomers

\begin{tabular}{cccccc}
\hline & $\begin{array}{c}\mathrm{B}_{40} \\
\text { monomer }\end{array}$ & $\begin{array}{c}\mathrm{B}_{40} \\
\text { dimer }\end{array}$ & $\begin{array}{c}\mathrm{B}_{40} \\
\text { trimer }\end{array}$ & $\begin{array}{c}\mathrm{B}_{40} \\
\text { tetramer }\end{array}$ & $\begin{array}{c}\mathrm{B}_{40} \\
\text { pentamer }\end{array}$ \\
\hline Gap & $3.18 \mathrm{eV}$ & $1.42 \mathrm{eV}$ & $1.10 \mathrm{eV}$ & $1.12 \mathrm{eV}$ & $1.06 \mathrm{eV}$ \\
\hline
\end{tabular}

principal quantum number of two. This decreasing trend is in agreement with a previous report [30]. Furthermore, we analyzed the HOMO-LUMO gap of the $\mathrm{B}_{40}$ tetramer and pentamer. We found that the HOMO-LUMO gap of the $\mathrm{B}_{40}$ oligomers did not continue to decrease as the $\mathrm{B}_{40}$ polymer size increased. When the $\mathrm{B}_{40}$ polymer size was three or more, the HOMO-LUMO gap was approximately $1.10 \mathrm{eV}$ and did not decrease further, as shown in Table 1. Table S2 lists the HOMO-LUMO gaps of the eight isomers for $\mathrm{B}_{40}$ dimers, with the HOMO-LUMO gap in the vertex-vertex conformation being the largest and that in the $7_{3} \perp 7_{1}$ conformation being the smallest. The $7_{3} \perp 7_{1}$ MD shows that the HOMO-LUMO gap is temperature dependent, ranging from 1.42 to $0.84 \mathrm{eV}$. Therefore, we consider $\mathrm{B}_{40}$ a superatom building block for assembled materials that can reduce the band gap and achieve the transformation from insulator to semiconductor. This is similar to $\mathrm{C}_{60}$ polymers, whose HOMO-LUMO gap also decreases with increasing polymer size [43].

\section{CONCLUSIONS}

We first investigated the interaction between $\mathrm{B}_{40}$ superatoms to understand their potential cluster assembly better using the density functional theory calculations. Energy decomposition analysis indicates that the $B_{40}$ oligomers are dominated by the orbital interaction between two monomers. The electronic-structure analysis shows that the $B_{40}$ oligomers retain the inner shell superatomic orbital of $\mathrm{B}_{40}$ monomers; however, the valence shell superatomic orbitals disappear due to bonding and antibonding hybridization between superatoms. Furthermore, the band gap decreases from 3.18 to $1.10 \mathrm{eV}$ as the $\mathrm{B}_{40}$ polymer size increases. The band gap is approximately 
$1.10 \mathrm{eV}$ when the $\mathrm{B}_{40}$ polymer size is three or more. $\mathrm{MD}$ simulations show that both the geometric and electronic structures of the $7_{3} \perp 7_{1}$ conformation are stable. Therefore, our research achieves the transformation from insulator to semiconductor via the $\mathrm{B}_{40}$ superatomic assembly, providing a new insight into the development of cluster-assembly materials.

\section{Received 13 June 2018; accepted 24 July 2018; published online 14 August 2018}

1 Kalsin AM, Fialkowski M, Paszewski M, et al. Electrostatic selfassembly of binary nanoparticle crystals with a diamond-like lattice. Science, 2006, 312: 420-424

2 Sharma J, Chhabra R, Cheng A, et al. Control of self-assembly of DNA tubules through integration of gold nanoparticles. Science, 2009, 323: 112-116

3 Shevchenko EV, Talapin DV, Kotov NA, et al. Structural diversity in binary nanoparticle superlattices. Nature, 2006, 439: 55-59

4 Daniel MC, Astruc D. Gold nanoparticles: assembly, supramolecular chemistry, quantum-size-related properties, and applications toward biology, catalysis, and nanotechnology. Chem Rev, 2004, 104: 293-346

5 Claridge SA, Castleman Jr. AW, Khanna SN, et al. Cluster-assembled materials. ACS Nano, 2009, 3: 244-255

6 Qian M, Reber AC, Ugrinov A, et al. Cluster-assembled materials: toward nanomaterials with precise control over properties. ACS Nano, 2010, 4: 235-240

7 Zhang S, Zhang Y, Huang S, et al. Theoretical investigation of growth, stability, and electronic properties of beaded $\mathrm{ZnO}$ nanoclusters. J Mater Chem, 2011, 21: 16905

8 Zhang S, Zhang Y, Huang S, et al. Theoretical investigations of sp$\mathrm{sp}^{2}$ hybridized zero-dimensional fullerenynes. Nanoscale, 2012, 4: 2839-2842

9 Roy X, Lee CH, Crowther AC, et al. Nanoscale atoms in solid-state chemistry. Science, 2013, 341: 157-160

10 Krätschmer W, Lamb LD, Fostiropoulos $\mathrm{K}$, et al. Solid $\mathrm{C}_{60}$ : a new form of carbon. Nature, 1990, 347: 354-358

11 Liu F, Mostoller M, Kaplan T, et al. Evidence for a new class of solids. First-principles study of $\mathrm{K}\left(\mathrm{Al}_{13}\right)$. Chem Phys Lett, 1996, 248: 213-217

12 Reber AC, Khanna SN, Castleman AW. Superatom compounds, clusters, and assemblies: ultra alkali motifs and architectures. J Am Chem Soc, 2007, 129: 10189-10194

13 Khanna SN, Jena P. Assembling crystals from clusters. Phys Rev Lett, 1992, 69: 1664-1667

14 Castleman AW, Khanna SN, Sen A, et al. From designer clusters to synthetic crystalline nanoassemblies. Nano Lett, 2007, 7: 27342741

15 Yang $\mathrm{H}$, Wang $\mathrm{Y}$, Huang $\mathrm{H}$, et al. All-thiol-stabilized $\mathrm{Ag}_{44}$ and $\mathrm{Au}_{12} \mathrm{Ag}_{32}$ nanoparticles with single-crystal structures. Nat Commun, 2013, 4: 2422

16 Champsaur AM, Yu J, Roy X, et al. Two-dimensional nanosheets from redox-active superatoms. ACS Cent Sci, 2017, 3: 1050-1055

17 Wang J, Yu T, Gao Y, et al. All-boron fullerene $\mathrm{B}_{40}$ : a superatomic structure. Sci China Mater, 2017, 60: 1264-1268

$18 \mathrm{Yu}$ T, Gao Y, Xu D, et al. Actinide endohedral boron clusters: A

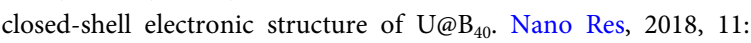

354-359

19 Zhai HJ, Zhao YF, Li WL, et al. Observation of an all-boron fullerene. Nat Chem, 2014, 6: 727-731

20 Jin $\mathrm{P}$, Hou $\mathrm{Q}$, Tang $\mathrm{C}$, et al. Computational investigation on the

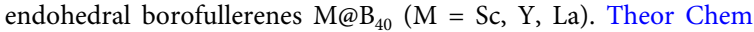
Acc, 2015, 134: 13

21 Bai $\mathrm{H}$, Chen $\mathrm{Q}$, Zhai $\mathrm{HJ}$, et al. Endohedral and exohedral me-

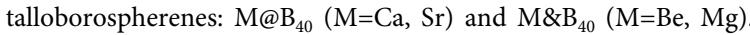
Angew Chem Int Ed, 2015, 54: 941-945

22 Fa W, Chen S, Pande S, et al. Stability of metal-encapsulating boron fullerene $\mathrm{B}_{40}$. J Phys Chem A, 2015, 119: 11208-11214

23 Dong $\mathrm{H}$, Lin B, Gilmore K, et al. $\mathrm{B}_{40}$ fullerene: An efficient material for $\mathrm{CO}_{2}$ capture, storage and separation. Curr Appl Phys, 2015, 15: 1084-1089

24 Gao G, Ma F, Jiao Y, et al. Modelling $\mathrm{CO}_{2}$ adsorption and separation on experimentally-realized $\mathrm{B}_{40}$ fullerene. Comput Mater Sci, 2015, 108: 38-41

25 Dong $\mathrm{H}$, Hou T, Lee ST, et al. New Ti-decorated $\mathrm{B}_{40}$ fullerene as a promising hydrogen storage material. Sci Rep, 2015, 5: 9952

26 Lin $\mathrm{B}$, Dong $\mathrm{H}, \mathrm{Du} \mathrm{C}$, et al. $\mathrm{B}_{40}$ fullerene as a highly sensitive molecular device for $\mathrm{NH}_{3}$ detection at low bias: a first-principles study. Nanotechnology, 2016, 27: 075501

27 Yang Z, Ji YL, Lan G, et al. Design molecular rectifier and photodetector with all-boron fullerene. Solid State Commun, 2015, 217: $38-42$

28 Shakerzadeh E, Biglari Z, Tahmasebi E. $M @ \mathrm{~B}_{40}(\mathrm{M}=\mathrm{Li}, \mathrm{Na}, \mathrm{K})$ serving as a potential promising novel NLO nanomaterial. Chem Phys Lett, 2016, 654: 76-80

29 Li Z, Yu G, Zhang X, et al. Bonding the superalkali $\mathrm{M}_{3} \mathrm{O}(\mathrm{M}=\mathrm{Li}$ and $\mathrm{K}$ ): An effective strategy to improve the electronic and nonlinear optical properties of the inorganic $\mathrm{B}_{40}$ nanocage. Physica $\mathrm{E}$ Low-dimensional Syst Nanostruct, 2017, 94: 204-210

30 Yang $\mathrm{Y}$, Zhang Z, Penev ES, et al. $\mathrm{B}_{40}$ cluster stability, reactivity, and its planar structural precursor. Nanoscale, 2017, 9: 1805-1810

31 Zheludev NI. The road ahead for metamaterials. Science, 2010, 328: $582-583$

32 Grimme S, Antony J, Ehrlich S, et al. A consistent and accurate $a b$ initio parametrization of density functional dispersion correction (DFT-D) for the 94 elements H-Pu. J Chem Phys, 2010, 132: 154104-154104

33 Adamo C, Barone V. Toward reliable density functional methods without adjustable parameters: The PBE0 model. J Chem Phys, 1999, 110: 6158-6170

34 Perdew JP, Burke K, Ernzerhof M. Generalized gradient approximation made simple. Phys Rev Lett, 1996, 77: 3865-3868

35 Hehre WJ, Ditchfield R, Pople JA. Self-consistent molecular orbital methods. XII. Further extensions of Gaussian-type basis sets for use in molecular orbital studies of organic molecules. J Chem Phys, 1972, 56: 2257-2261

36 He R, Zeng XC. Electronic structures and electronic spectra of allboron fullerene $\mathrm{B}_{40}$. Chem Commun, 2015, 51: 3185-3188

37 Frisch MJ, Trucks GW, Schlegel HB, et al. Gaussian 09, revision d.01. 2013

38 Morokuma K. Molecular orbital studies of hydrogen bonds. III. $\mathrm{C}=\mathrm{O} \cdots \mathrm{H}-\mathrm{O}$ hydrogen bond in $\mathrm{H}_{2} \mathrm{CO} \cdots \mathrm{H}_{2} \mathrm{O}$ and $\mathrm{H}_{2} \mathrm{CO} \cdots 2 \mathrm{H}_{2} \mathrm{O}$. J Chem Phys, 1971, 55: 1236-1244

39 Ziegler T, Rauk A. On the calculation of bonding energies by the Hartree Fock Slater method. Theoret Chim Acta, 1977, 46: 1-10

40 te Velde G, Bickelhaupt FM, Baerends EJ, et al. Chemistry with ADF. J Comput Chem, 2001, 22: 931-967 
41

Delley B. From molecules to solids with the $\mathrm{DMol}_{3}$ approach. J

Chem Phys, 2000, 113: 7756-7764

42 Kurakevych OO, Solozhenko VL. Rhombohedral boron subnitride, $\mathrm{B}_{13} \mathrm{~N}_{2}$, by X-ray powder diffraction. Acta Crystlogr C Cryst Struct Commun, 2007, 63: i80-i82

43 Beu TA, Onoe J, Hida A. First-principles calculations of the electronic structure of one-dimensional $\mathrm{C}_{60}$ polymers. Phys Rev $\mathrm{B}$, 2005, 72: 155416

Acknowledgements This work was supported by the National Natural Science Foundation of China (11674123 and 11374004). Wang Z also acknowledges the High Performance Computing Center of Jilin Uni- versity.

Author contributions Wang $\mathrm{Z}$ proposed the project; Wang J calculated and analyzed the results. All authors contributed to the general discussion.

Conflict of interest The authors declare no conflict of interest.

Supplementary information The total energy for the eight isomers, bonding energy decomposition details, molecular orbitals for the $\mathrm{B}_{40}$ oligomers, DOS, and HOMO-LUMO gap for the eight isomers are available in the online version of the paper.

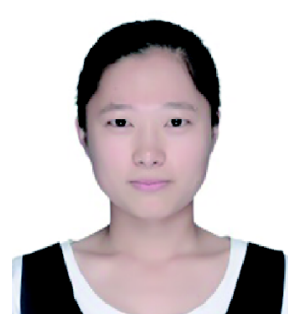

Jia Wang is an $\mathrm{PhD}$ candidate in prof. Zhigang Wang's group at the Institute of Atomic and Molecular Physics, Jilin University. Her current research focuses on the spin polarization effect of p electrons.

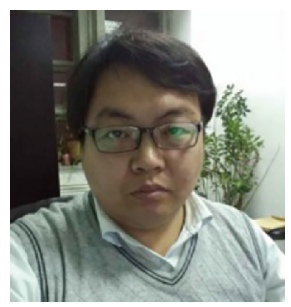

Zhigang Wang received his BSc degree from the College of Physics, Jilin University in 2001 and his PhD degree from the Institute of Atomic and Molecular Physics, Jilin University in 2006, under the supervision of Prof. Shoufu Pan. He joined the Institute of Atomic and Molecular Physics, Jilin University in 2011 as a full professor. His research interests focus on the intermolecular interactions of nanomaterials.

\section{超原子组装诱导的从绝缘体到半导体性质转变: 一个理论研究}

$$
\text { 王佳 }{ }^{1,2} \text {, 姜万润 }{ }^{1,2} \text {, 解伟誉 }{ }^{1,2} \text {, 王健鹏 }{ }^{1,2} \text {, 王志刚 }{ }^{1,2^{*}}
$$

摘要 嗍基超原子通向功能材料的一个途径是组装. 我们通过密度泛函理论研究了典型的嗍基超原子 $\mathrm{B}_{40}$ 之间的相互作用. 结果显示, 在不 同的低聚物中两个 $\mathrm{B}_{40}$ 之间不同的朝向方式会导致电子结构改变, 但它们都部分保持了超原子性质. 这是因为单体中靠内壳层的超原子轨 道仍保持其在超原子中的电子局域性，而价壳层的超原子轨道由于超原子间成键或反键杂化而不能保持孤立超原子的轨道形状. 在部分 保持超原子性质的情况下, $\mathrm{B}_{40}$ 超原子的组装可以相应实现从绝缘体到半导体的转变. 带隙的减小是由“主量子数”为 2 的超原子轨道杂化成 键导致的. 我们的发现凸显了超原子间相互作用, 会带来不同于单体的协同效应. 因此, 这一研究将有助于新型材料和器件的发展, 尤其在 以超原子为功能单元的组装材料研究方面将发挥积极作用. 